\title{
XVIII.
}

\section{Vergessene Physiognomonika.}

In der sorgfältigen 'Sylloge locorum physiognomonicorum', die Förster im zweiten Bande seiner 'Scriptores physiognomonici' p. 233 sq. zusammengestellt hat, fehlen einige Stellen, die mit dem Kaiser Julian in Beziehung stehen und teils für die Kenntnis seiner Person, teils für die Erklärung seiner Schriften von Bedeutung sind:

A. Das Julianporträt bei Gregor von Nazianz').

In der zweiten Invektive sagt der Kirchenvater (c. 23 t. I p. $161 \mathrm{D}$ sq. ed. Maur.) im Anschluß an seine Begegnung mit dem Prinzen in Athen:

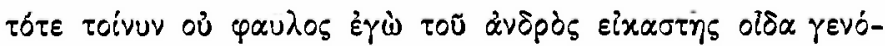

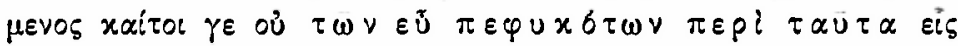

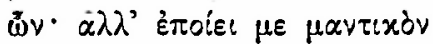

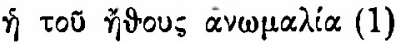

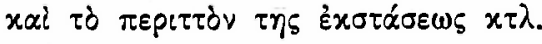

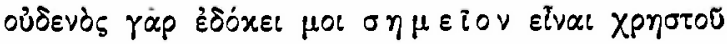

$\left.\alpha \dot{u} \times \grave{\eta} \nu \alpha \dot{\alpha} \pi \gamma \dot{\eta} \varsigma^{2}\right)(2)$

WH

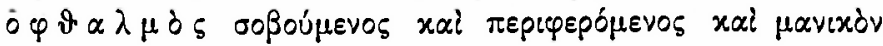

$\beta \lambda \dot{\varepsilon} \pi \omega \nu(4)$,

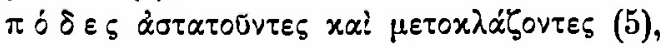

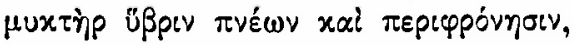

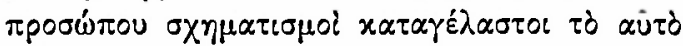

$\left.{ }^{1}\right)$ Schon angeführt von Orbilius Anthroposcopus (J. G. Fr. Franz), Versuch einer Geschichte der Physiognomonik und der damit verbundenen Wissenschaften. Wien und Leipzig 1784 S. 238.

2) In der Historia tripartita (t. 69 col. $1065 \mathrm{~B}$ bei Migne) ist hieraus cervix inflexibilis geworden. 


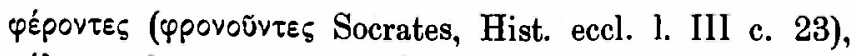

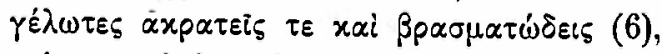

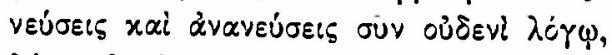

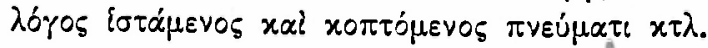

Während Gregorius Presbyter in seiner Vita des Nazianzeners (a. a. 0. p. CXXXVII) diesen Passus bloß ungenau paraphrasiert, weist Basilius Minimus (t. 36 col. $1141 \mathrm{~A}$ bei Migne) ganz deutlich auf seinen physiognomonischen Charakter hin. indem er von dem j̃

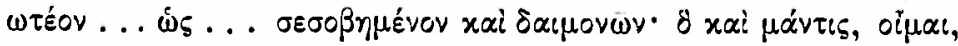

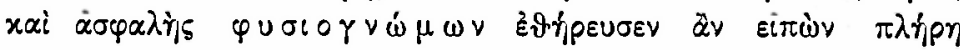

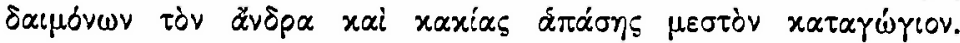
Die $\varepsilon \tilde{u} \pi \varepsilon \varphi u x \dot{\delta} \tau \varepsilon \varsigma \pi \varepsilon \rho l \tau$ $\tau \tilde{0} \tau \alpha$, zu welchen Gregor sich bescheidener Weise nicht rechnet, sind also diesem Scholion zufolge

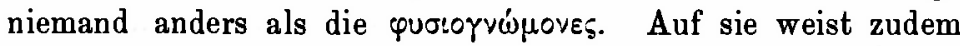

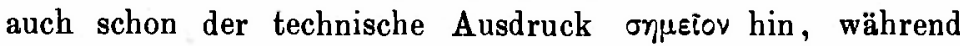
andrerseits bei der Aufzählung der äußeren Merkmale der Mangel einer sachgemäßen Reihenfolge und die willkürliche Beifügung oder Weglassung einer entsprechenden Deutung den Laien verraten. So wenig Verlaß nun auch auf die sehr subjektive Pseudowissenschaft der Physiognomonik im allgemeinen ist, so gerne möchte man doch gerade bei den

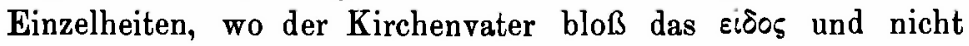
zugleich auch das sich daraus ergebende $\gamma_{i}^{*} \circ 05$ charakterisiert, sich eine Vorstellung von dem Eindruck bilden, den diese Züge nach Maßgabe der Physiognomoniker auf ihn machen konnten. Diese letzteren geben nun leider nicht an allen, sondern nur an den von uns durch beigeschriebene Ordnungszahlen gekennzeichneten Stellen Bescheid. Zudem kommen bei ihnen die von Gregor hervorgehobenen Merkmale in den meisten Fällen nicht absolut, sondern nur in Verbindung mit andern vor, von deren Vorhandensein oder Fehlen wir bei der Persönlichkeit Julians keine genauere Kenntnis haben. Da der Nazianzener von vornherein jeweils nur auf ein schlim-

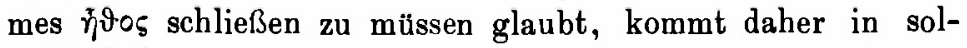
chen Fällen, wo die julianischen Merkmale in irgend einem zusammengesetzten Typus anch eine vorteilhafte Auslegung zulassen, dieses eióos nicht in Betracht. Unter diesen die $\mathrm{Zu}-$ 
verlässigkeit des Resultates sehr beeinträchtigenden Einschränkungen ist folgendes aus den Physiognomonikern zu entnehmen:

Zu 1: Physiognomonica Pseudaristotelis (vol. 1) p. 28,8

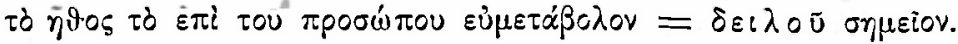

$\mathrm{Zu} 2$ (vgl. Ammian. 1. XXV c. 4, 22 von Julian: opima et incurva cervice): Physiognomonica Polemonis ${ }^{3}$ ) arabice (vol. I) p. 258,15 cervicum laxitas signum adfectationis vonseiten eines Stärke heuchelnden $\mathrm{Schwächlings} \mathrm{(vgl.} \mathrm{a.}$ a. 0 . p. 258, 9 sunt quoque, qui eo gressum augeant und Ammian. l. XXII c. 14,3 ridebatur ... ut homo brevis, grandia incedens). - Physiognomonica Adamantii (vol. I) p. 367,3

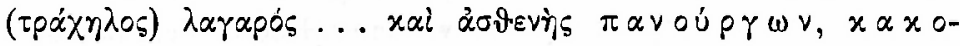

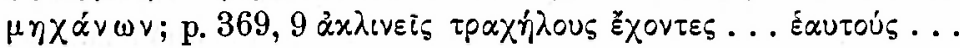

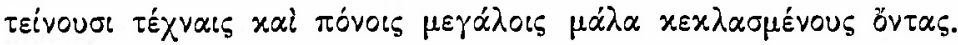

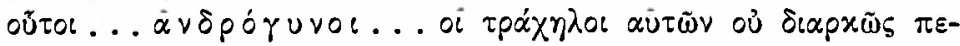

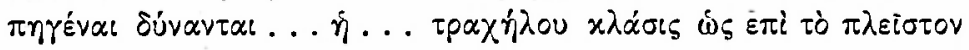

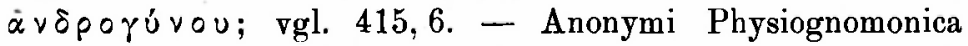
graec. (Vol. II) p. 228, 21 sq. - Fragmenta (vol. II) p. 276, 7; 306, 17; 307, 29. - Physiognomonica Anonymi latine (vol. II) p. 58,5 cervix tenuis $=$ indicinm eines Eu nuchen; p. 72,10 cervix ... tenuis timiditatem et malignitatem declarat; p. 73, 1 cervix ... soluta et invalida ... nocentes et insidiosos homines praedicat; p. 76, 7 infracta . . . cervix = Zeichen eines (homo) vel stultus vel effeminatus. - Fragm. p. 299, 26 àvíp . .

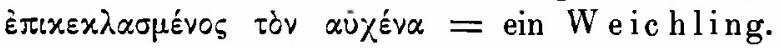

Zu 3 (vgl. Ammian. 1. XXII c. 14,3 videbatur ... ut homo brevis, humeros extentans): Pseudarist. p. 89, 9 oi . . .

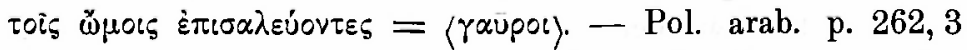
si . . u umeros vides moventem, ... ei tumultuationem et audaciam, dum aliquid molitur, et homines consulendi evitationem adiudica. - Physiognomonica Adamantii (vol. I) p. 400,2 $\delta$

8) Daß Gregor die Physiognomonik des Polemo (vgl. Foerster a. a. O. vol I Proleg. p. LXXV sq. und desselben Dissertatio de Polemonis physiognomonicis Kiliae 18r6) selbst gelesen hätte, ist nicht

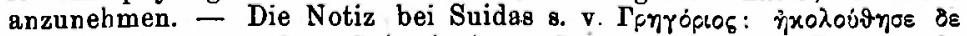

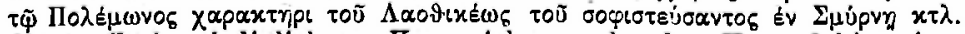
dient offenbar lediglich zur Kennzeichnung des dem Kappodokier eige. nen Stils. 


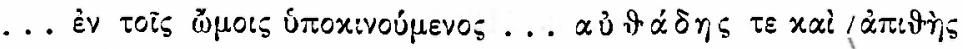
xai) ußprotís. - Anonym. lat. p. 100,2 qui ... humeros commovet ... superbus atque insolens; p. 130,1 humeros eidem (sc. einem homo sceleratissimus) ... spasmus frequen-

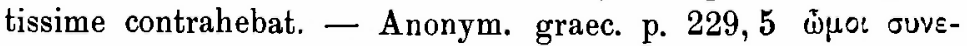

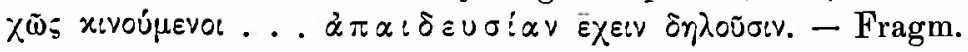
p. 330,1 umerorum ... allevatio atque contractio ... gestum quendam humilem atque servilem et quasi fraudulentum facit (vgl. Cicero, Brutus 60 C. Julius ... ex ... in utramque partem toto corpore vacillante quaesivit, quis loqueretur e lintre ${ }^{4}$ ).

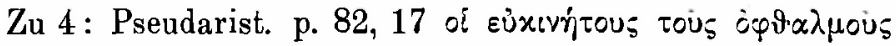

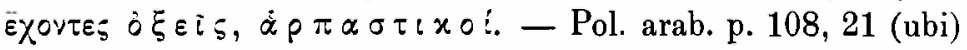
vides ... oculos concitatos, in ipsis valde mobiles..., eo scias iam esse potitum daemonem (vgl. Gregor. Naz. or. IV

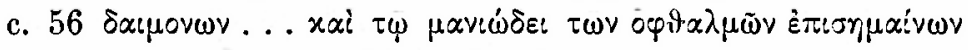

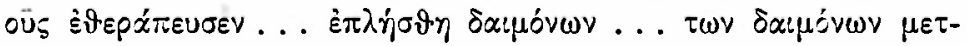
$\varepsilon: \lambda \eta \varphi \omega \omega_{s}$ u. o. das Scholion des Basilius Minimus) insolentissimum, iracundum, vindictae cupidum, qui in eum fugacissimum tentationes immittat. Si vero perpetuo circumvolvuntur, eorum possessor facinus foedum patravit, ut cognatorum caedem aut rei vetitae persecutionem ex iis, quas deus summus detestatur, instar istius, quod Pelopis filius perpetravit, qui filium epulans inventus est, vel ut Oedipus, Lai filius, qui corpus cum matre miscuisse fertur . . . Talis est etiam Thraciae regionis ad Constantinopolim pertinentis regionis incolarum de-

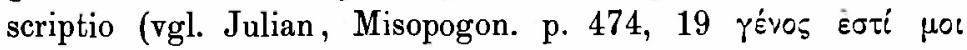

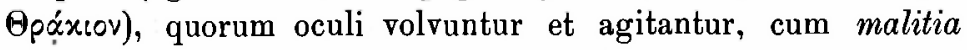
incliti sint, sed a perpetrandis malis vehementi metu et timore retineantur, quamquam studium eorum perpetuo in malum propensum est; p. 114, $9 \mathrm{Ubi}$ in oculis vides agitatas et palpebras et interiorem partem eorum, possessori eorum timiditatem adiudicato; p. 114, 21 Oculus nictans vel tremens ..., eius possessori segnitiem, pigritiam, apoplexiam, stuprum, vino-

4) Diese Stelle meint wohl Montacutius, wenn er in seiner Separatausgabe der beiden Invektiven (Etonae 1610) das 'ciceronische' gestum agere humeris et quodammodo de lintre loqui exserto humero et rursum ad aequilibrium depresso zum Vergleiche bezieht. 
lentiam adiudicato etc.; p. 116, 12 Ubi oculum vides totum agitatum, tamquam festuca insit ei, scias eius possessorem rei venereae valde deditum esse et lusum et cuppedia amare; p. 124, 3 sq.; p. 144, 17 Oculum ... valde mobilem videns ei dolum tribuas et astutiam et pravam cupidinem; p. 156, 17 Quodsi ... oculo .... inesse vides tremorem ...., adscribe eis insaniam. Adfectos enim insania aut adfectum iri eos recte reperies; p. 164, 8 Oculi, qui perpetuo aperiuntur et conivent, timiditatem indicant; p. 242,19 oculi . . . valde mobiles $=$ typus Graecus purus; p. 260, $17 \mathrm{Si}$ cum gressus celeritate oculorum mobilitatem faciliorem et perturbationem coniungit et in capite movendo et in vehementer anhelando multus est: haec proprietas est hominis acris, conscii mali, quod perpetravit, facinoris; p. 270, 13 viri timidi signum, id est imparis et imbecillis, illud est eum ... esse oculorum palpebris crebro motis, respiratione vehementi etc.; p. 276, 15 signum (androgyni effeminati; vgl. p. 123,5 sq.) est, ut videas eum . . oculis valde fulgurantem et eos agitantem. - Vgl. Adam. p. 306, 8 sq.; Anonym. lat. p. 33, 2 sq.; Pseudopol. ar. (vol. II) p. 152, 1 sq.; Rasis (vol. II) p. 164, 15 sq.; Secreti secret. (vol. II) p. 201, 2; Anonym. graec. p. 226, 5 ; Fragm. p. 278, $21 ; 299,14 ; 308,14$.

Zu 5: Pol. arab. p. 282, 3 saepe pedibus suis terram calcat et ferit, celeri motu = signum viri amari animi. Vgl. Adam. p. 417, 3. Anonym. lat. p. p. 124, 10; 123, 9 (De androgyno) ... cuius omnes artus incerti status sunt, qui insilit frequenter imis pedibus et calcaneis saepius insurgit, qui genua collidit. Vgl. Pol. arab. p. 262, 19; 276, 18; 280, 12 ; Adam. p. 416, 1. - Pol. arab. p. 284, 11 pedes eius (sc. cui malum et afflictio imminet inscio) inter eundum iactabantur .. . quae signa nisi in insano non videbis, immo vero ei malum adfore certo iudicabis; Fragm. p. 237, $9=$ Hom. Il. v,

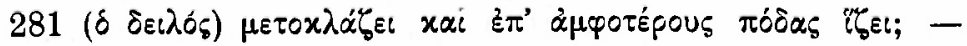
Pol. arab. p. 204, 20 genua laxa, mollia, instabilia . . . e signis mulierum et effeminationis sunt.

Zu 6: Pol. arab. p. 128, 8 Quotienscunque (homo mali plenus) ridebat, alta voce ridebat; ... eum observabam risu correptum; vgl. p. 148, 21; 150,9 nec nisi ridens invenie- 
batur (homo turpis); - Pseudopol. arab. p. 154, 14 qui magnum ridet, impudens est et cavillator. - Rasis p. 170, 6. -

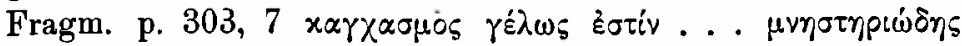

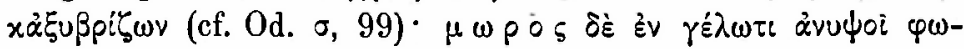
vìv auтoũ (= Sirac. 21,20); p. 305, 27 (= Sirac. ${ }^{5}$ ) 19, 30

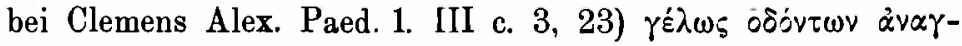

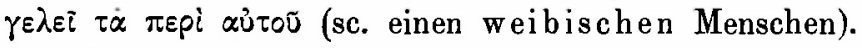

Faßt man diese mannigfaltigen $\ddot{\eta} \vartheta \eta$, von denen manche sich in den von physiognomonischen Einflüssen unabhängigen Partien der Invektiven wiederfinden, zu einem Gesamtbild zusammen, so muß Julian auf Gregor, wenn dieser auch nur eine ungefähre und allgemeine Kenntnis von den Aufstellungen der Physiognomoniker besaß, diesen zufolge den Eindruck eines weichlichen, affektierten, bösartigen und tückischen Menschen gemacht haben, in dessen unmännlichem Wesen etwas unbestimmbar Dämonisches lag.

Diese Charakteristik wird durch eine gleichfalls physiognomonische Stelle bei A m mian ergänzt. In dessen Julianporträt

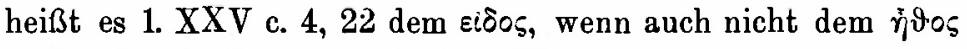
nach übereinstimmend mit dem Nazianzener: venustate oculorum micantium flagrans (vgl. Mamertinus, Gratiarum act. c. 6: micantia sidereis ignibus lumina), qui mentis eius angustias ${ }^{6}$ ) indicabant. Vgl. Anonym. lat. p. 50, 3 trementes micantesque oculi et salientes, quos Graeci $\pi \alpha \lambda \lambda$ opéveus dicunt, ... magnarum rerum cogitatorem ... indicant... his oculis aestimatur etiam Alexander Magnus fuisse. - Adam. p. 328, 1

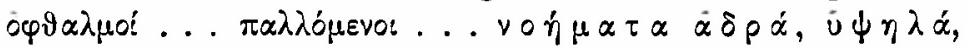

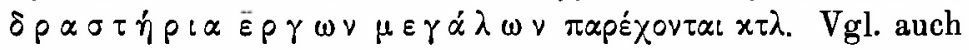
Anonym. lat. p. 57, 13 oculi ... micantes leniter intendentes tamquam concinnati ad suavitatem et gratiam, wo leider gerade die Deutung ausgefallen ist.

B. Der liebeskranke Antiochus.

In seinem Misopogon erzählt Julian p. 447, 8 sq. ed.

$\left.{ }^{5}\right) \mathrm{Zu}$ der ganzen Stelle vergleicht schon Clemencet unmittelbar Eccl. XIX 27 Amictus corporis, et risus dentium, et ingressus hominis enuntiant de illo.

6) Von Wagner (zu der Stelle t. III p. 106) richtig durch ár $\chi^{i v o l \alpha}$ erklärt. 
Hertlein die auch aus Suidas, Valerius Maximus, Plutarch, Lucian u. a. bekannte Geschichte von der heimlichen Liebe des Prinzen Antiochus von Syrien zu seiner Stiefmutter Stratonike ${ }^{7}$ ). In der von dem Kaiser vorgetragenen Version dieser von Galen, De praecogn. (vol. XVIII p. 18 ed. Kühn) aus-

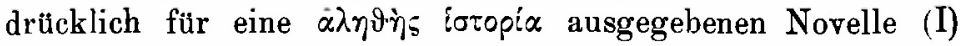
wird p. 447, 15 sq. der Zustand des Liebeskranken mit folgenden Worten geschildert:

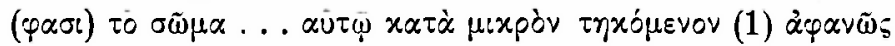

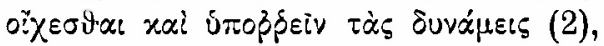

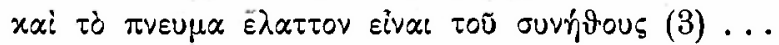

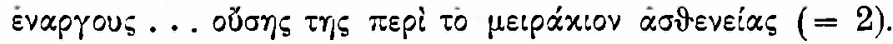

Sodann werden von dem Arzte Erasistratos bei der $9 \eta^{\prime}-$

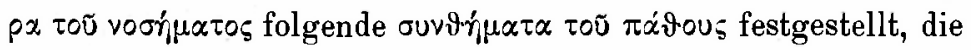
sich beim Erscheinen der Geliebten zeigen:

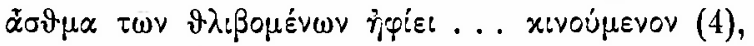

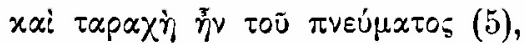

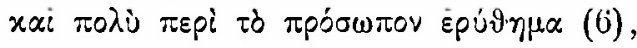

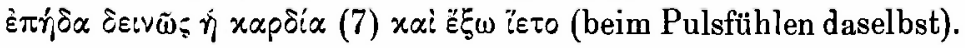

Diese Symptome dienen jenem allerdings hier mehr zur Ermittelung der geliebten Persönlichkeit als zur Feststellung des Leidens an sich. Diesem war er schon vorher auf die

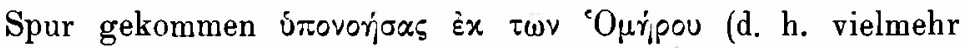

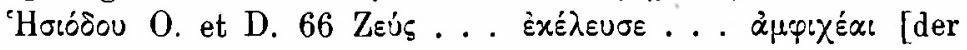

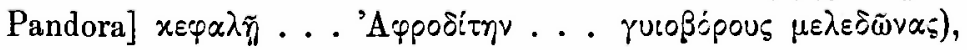

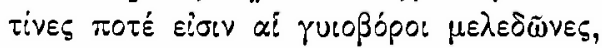

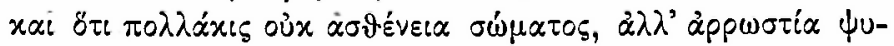

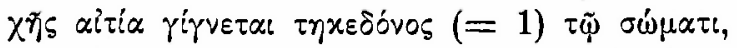

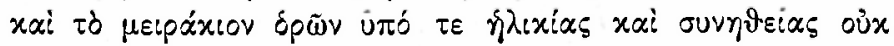

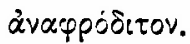

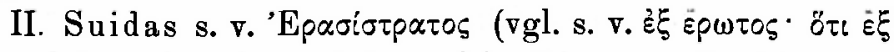

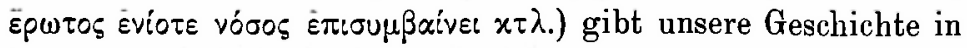

$\left.{ }^{7}\right)$ S. Rohde ${ }^{2}$. Der griechische Roman S. $55 \mathrm{ff}$.; Rosenbaum in der Allg. Enc. der Wissenschaften Sect. 1. Th. 36 S. 151 u. Erasistratus) und Fuchs, Erasistratea, quae in librorum memoria latent, congesta enarrantur. Diss. Berol. 1692 p. 12,23. - Unter dem physiognomonischen Gesichtspunkt besprechen den Fall schon Scipio Claramontanus, De coniectandis cuiusque moribus et latitantibus animi affectibus libri $\mathrm{X}$ Lugduni 1704 p. 652.661 und Orbilius Anthroposcopus a. a. O. S. 148. 
verkürzter Form dem Resultat nach wieder und behauptet, der

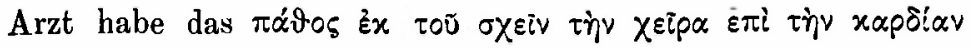

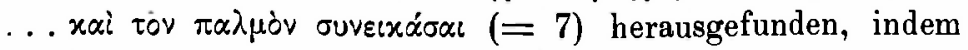

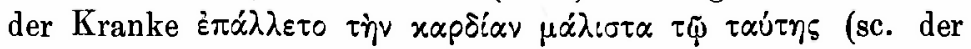

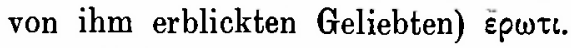

III. Bei Valerius Maximus l. V c. 7 Ext. 1 zeigt der Liebeskranke ultimam tabem (=1), und der Entdecker seines Leidens (hier der mathematicus Leptines bzw. nach anderer Version Erasistratos) bemerkt an ihm ad introitum Stratonices: rubore perfundi $(=6)$

et spiritu increbrescere $(=5)$ eaque egrediente pallere (8) et excitatiorem anhelitum subinde recuperare (vgl. 3, 4) und wird

brachium adulescentis ... apprehendendo modo vegetiore modo languidiore pulsu venarum $(7 \mathrm{a})$ auf die richtige Fährte gebracht.

IV. A p p i a n. bell. Syr. $\$ 59$ berichtet, Erasistratos habe

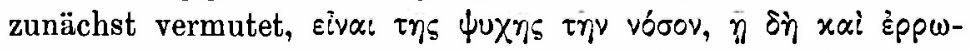

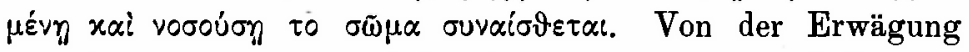
ausgehend, daßs namentlich die Liebe geheim gehalten zu

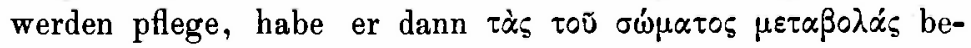

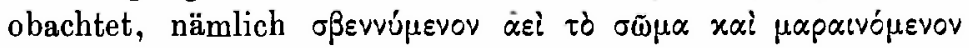

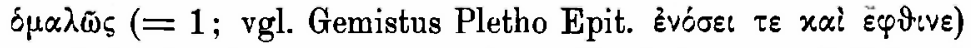

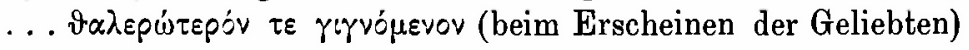

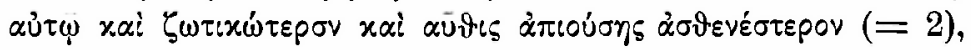
und so die Wahrheit herausgebracht.

V. Nach Pl u ta r ch, Demetrius 38 erkannte Erasistratos mit leichter Mühe die Liebe als Krankheitsursache. Er wendete, um die geliebte Persönlichkeit zu ermitteln, seine Aufmerksamkeit $\tau \bar{\varphi} \pi \rho \sigma \sigma \dot{\omega} \pi \omega$ des Kranken zu und beobachtete $\tau \dot{\alpha}$

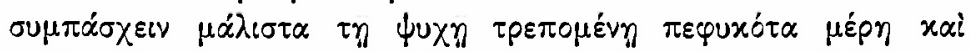

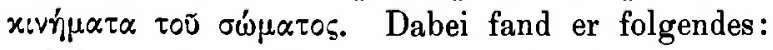

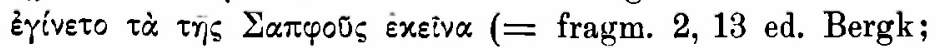

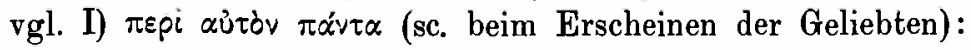
$\varphi \omega v \tilde{\eta}_{5}$ Ė $\pi i \sigma \chi \varepsilon \sigma l \zeta$ (vgl. 4),

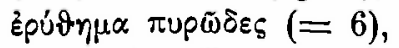

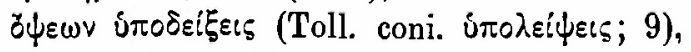

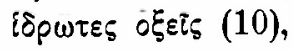

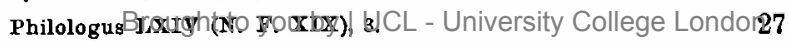




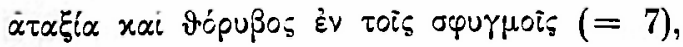

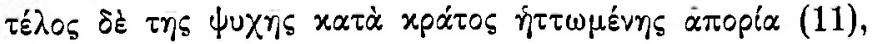

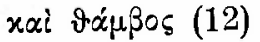

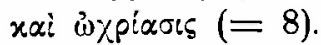

VI. Bei Pseudo-L u ci an, De dea Syria 17 heißt es von dem Patienten:

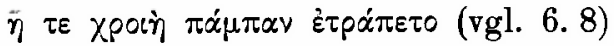

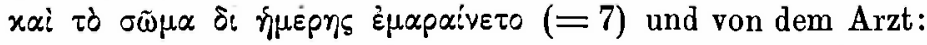

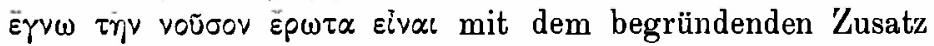

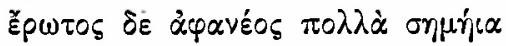

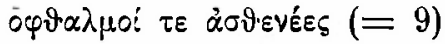

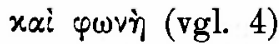

xaì xpoìे (vgl. 6. 8)

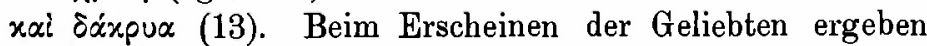
sich folgende Symptome:

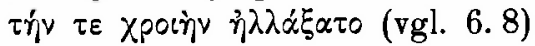

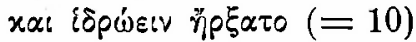

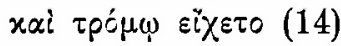

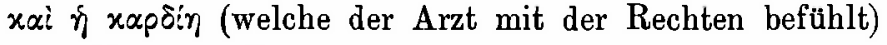
$\dot{\alpha} \vee \varepsilon \pi \dot{\alpha} \lambda \lambda \varepsilon \tau \circ$ (=7).

VII. Der von Galen a. a. 0. p. 40 mitgeteilten Version der sophistischen Aerzte zufolge brachte Erasistratos das Leiden

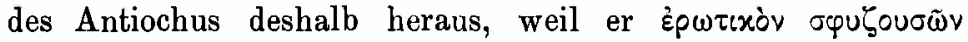

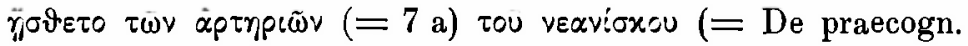
vol. XIV p. 613; vgl. Tzetzes, Chil. VII Hist. 118, 172 นóv

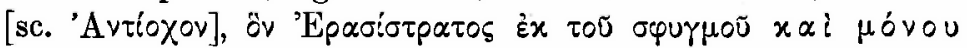

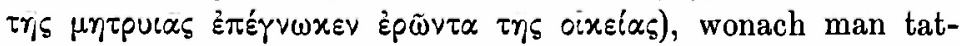

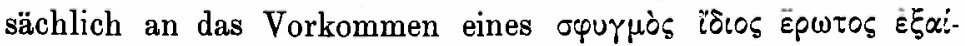

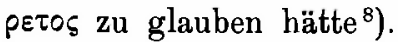

Solche absolute und ausschließliche Liebes- $\sigma \eta \mu \varepsilon i \alpha$, deren Existenz ja auch Lucian a. a. 0 . behauptet, gibt es nun auch nach der Theorie der Physiognom oniker. Es kommt hier folgendes in Betracht:

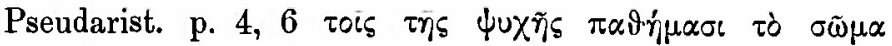

$\left.{ }^{8}\right)$ Hierüber vgl. die von Orbilius a. a. S. 145 zitierte Abhandlung Christoph. Blitzeri a Bilitz resolutio quaestionis: an pulsus aliquis amatorius concedendus in Greg. Horstii Dissert. de natura amoris, Giessae 1611. $4^{\circ}$, fol. F sq., wo der Fall des Antiochus fol. F 2 behandelt wird. 


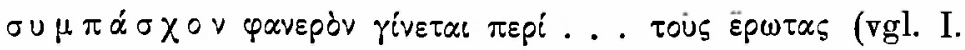

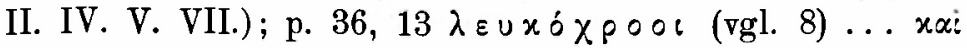

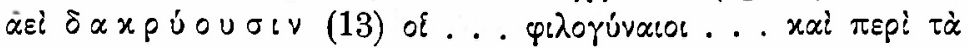

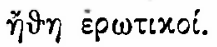

Arab. p. 106, 14 Caput LXIX: De signo mulierum erga quemvis peregrinum amoris $=$ p. 286, 1 sq.: Ein Fall, wo die geliebte Persönlichkeit anwesend ist; 25 signa; p. 288, 5 (beim liebenden Jüngling:) oculis aqua mersis $(=13)$... spiritum altum (vgl. 3. $4^{9}$ ) .. vestes sudore madefactas $(=10$ ) ... nasum palpitantem (vgl. 14) et colorem eius . . modo pallore $(=8)$ modo rubedine $(=6)$ variegatum ... tremore tenebatur (14); p. 288, 11 (bei der liebenden Frau:) oculi aqua mersi $(=13)$.

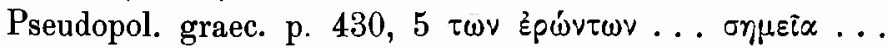

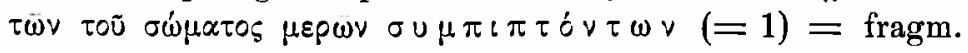
p. 308, 15 (= Oribas. Synops. VIII, 9 bzw. Paulus Aegin. III, 17).

Anonym. lat. p. 133, 6 amoribus ... deditus ... debet ... oculos habere prope lacrimosos $(=13$ ) et prope pallidos $(=8)$.

Fragm. p. 278, $23 \alpha i \ldots \chi p o \alpha i \alpha u ̉ \tau \tilde{\omega} v$ (sc. der Liebenden) 'ं) $\chi \rho \propto \alpha^{\prime}(=8)$.

Die Uebereinstimmung dieser technischen $\sigma \eta \mu \varepsilon i \alpha$ mit den novellistischen fällt sofort in die Augen. Galen vermehrt unser Material noch durch zwei Fälle aus seiner eigenen Praxis: Bei einem liebeskranken Jüngling (a. a. O. vol. XVIII B p. 40)

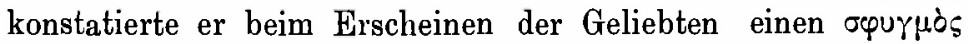

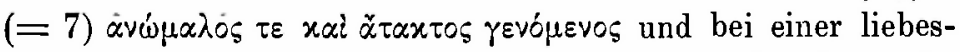
kranken Frau (a. a. O. vol. XIV p. 626, 631, 633 ${ }^{10}$ ) gibt er die bei der Erwähnung des Geliebten beobachteten Symptome

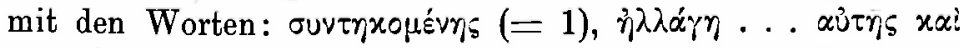

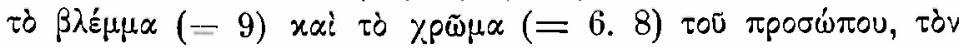

9) Hierdurch wird die Richtigkeit der Emendation excitatiorem (st. des handschriftlichen exercitatiorem) in der 0 . ausgeschriebenen Stelle des Valerius Maximus, wo Perizonius den anhelitus als einen „ex imo pectore petitum" erklärt, bestätigt. S. Kempfs Erklärung in seiner Ausgabe p. 449.

10) Schon angeführt von Scipio Claramontanus a. a. O. p. 715 und Orbilius Anthroposcopus a. a. S. 213. 


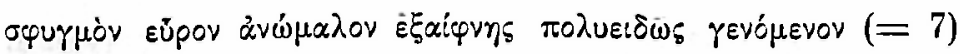
wieder, und zwar beidemale mit der Versicherung, daß es einen besonderen erotischen Puls nicht gebe, sondern daß man aus einem beschleunigten Pulsschlag lediglich auf $\tau \alpha p \alpha \chi \omega \delta \varepsilon \varsigma$ t? $\pi \alpha \dot{\vartheta} \circ \varsigma$ in der Seele des Kranken schließen dürfe, und nur ein glücklicher Zufall, wie er bei ihm selbst und wohl auch bei Erasistratos gewaltet habe, zu einer richtigen Diagnose führen könne. Damit ist aber zugleich der physiognomonischen Diagno-

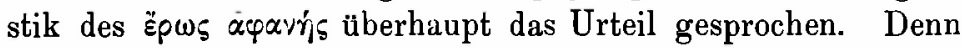
verfolgt man den Verlauf unserer Liebesgeschichte - unerklärlicher Zustand des Kranken, Vermutung eines psychischen Leidens, eventuell heimlicher Liebe, Beobachtung des Patienten in Anwesenheit einer liebenswerten Persönlichkeit, Feststellung einer starken seelischen Erregung angesichts derselben und Bestätigung der Annahme durch diesen Befund - so bleibt kein einziges unmittelbares Liebessymptom mehr übrig: Der Arzt hat keine andere Handhabe als eine völlig vage, auf seine Dichterlektüre, seine allgemeine pathologische Erfahrung und seine persönliche Kenntnis des Kranken gestützte Vermutung,

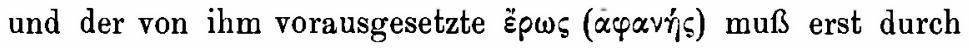

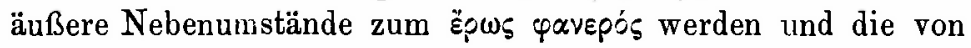
den Dichtern beobachteten Ausdrucksformen annehmen, damit die hypothetische Diagnose gerechtfertigt werden kann ${ }^{11}$ ).

Die Antiochus-Geschichte hat bereits ein älteres Vorbild in einer von Hippokrates an dem König Perdikkas vollzogenen Heilung ${ }^{12}$ ). Bei Soranus im Leben des Koers (vol. XXIII p. 851 ed. Kühn) heißt es: $\dot{\varepsilon} \propto \alpha \mu \alpha \dot{\sigma} \sigma \eta$ (sc. Hippocrates), $ّ \sigma \tau \varepsilon$

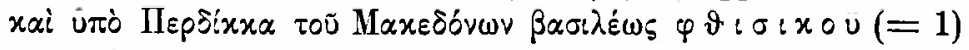

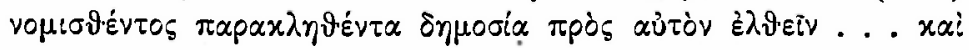

11) Blitzer entscheidet die Frage a. a. O. fol. F 2 v. u. im Sinne Galens mit folgenden Worten: Amorem omnino ex pulsu deprendi posse, si sit inconstans, varius, celer etc., praesertim si ad aspectum amasiae id contingat .. . In amore . . . nulla causa (inconstantiae etc.) vel evidens vel interna patet. Nequaquam tamen concedendum . . ut iste pulsus amatorius indigetetur, quasi ab aliis sit distinctus et infallibiliter hunc affectum detegat.

12) Bereits von Blitzer a. a. O. fol. F 2 o. angeführt. - Vgl. auch die von Eunapius, Vita Aedesii ed. Boissonade vol. I p. 38 erzählte

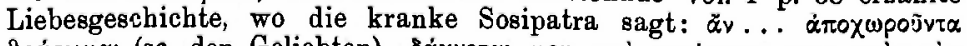

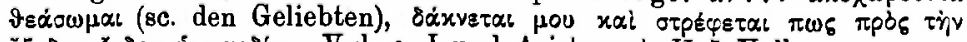

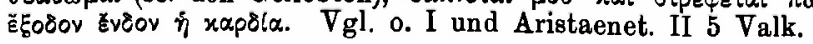




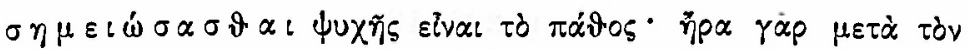

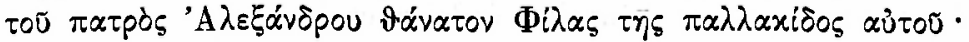

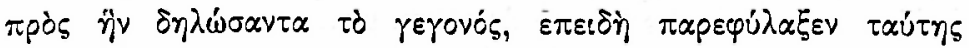

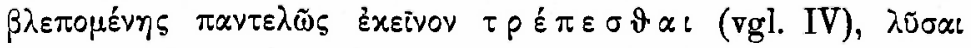

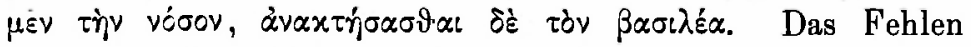
eines besonderen Liebeskriteriums stellt diese Anekdote in eine Reihe mit den nichtsophistischen Versionen der syrischen Erzählung und bestätigt so mittelbar die Richtigkeit der galenischen Kritik.

C. Diodorus von Antiochia.

Julian, Epist. 79 p. 606, 22 sagt von seinem asketischen Gegner Diodorus: ab ipsis punitur diis . . . in corruptionem thoracis incidens... Omne eius corpus consumptum est. Nam malae (I) eius conciderunt, rugae (II) vero in altitudinem corporis descenderunt. Quod non est philosophicae conversationis indicio, sicut videri vult a se deceptis . . . - faciem pallore (III) confectam (das Verbum fehlt). Was der Kaiser hier dem Diodorus abstreitet, nämlich das indicium philosophicae conversationis, gab es in der Tat nach der Lehre der Physiognomoniker. Pol, arab. p. 104, 21 verzeichnet ein ganzes (LV) Caput de signo viri litterarum et philosophiae amantis $(=$ p. 272, 1), worin es p. $272,7 \mathrm{u}$. a. heißt: nec multam in corpore pinguetudinem nec paucam habeat ${ }^{13}$ ). Da Julian uns den Eindruck verschweigt, den die äußeren Merkmale des Priesters auf ihn machten, so mögen die Physiognomoniker für ihn antworten:

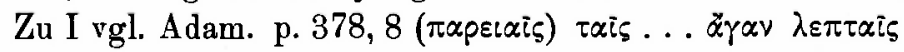

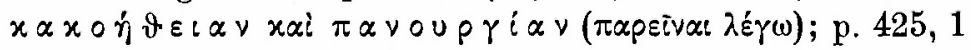

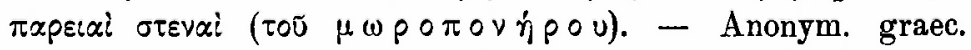

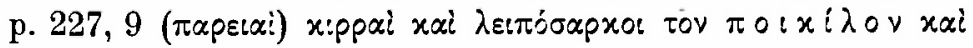

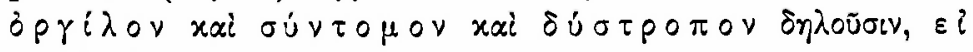

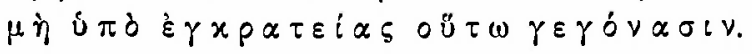

$\left.{ }^{13}\right)$ Vgl. die physiognomonische Beschreibung des Philosophen Isidorus bei Damascius $\$ 16$ ed. Westermann, wo es heißt: oi ... $\quad b_{\varphi} \vartheta \propto \alpha$ -

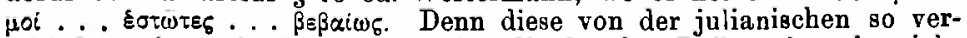
schiedene (s. o. A. 4) Augenart ist die für den Philosophen bezeich-

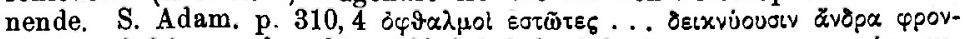

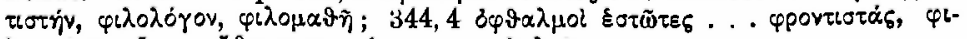

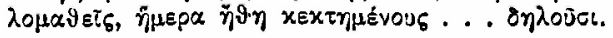


Zu II vgl. Pol. arab. p. 226, 13 Ubi in mala ... corrugatio est, eis fraudem malam indolem et vilitatem animi tribue, nam suibus similes sunt. - Anonym. graec. p. 227, $13 \pi \alpha \rho \varepsilon\llcorner\alpha$

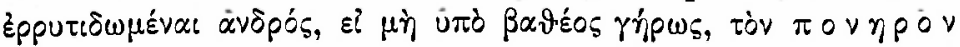

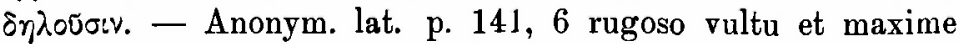
malis. huismodi mulier inepta, inefficax, ingrata. - Pseudarist.

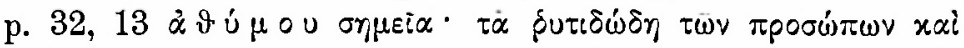

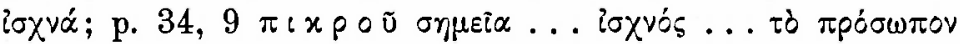

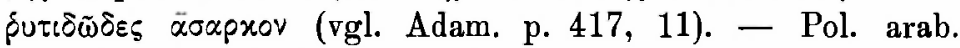
p. 114, 2 (si) frontem vides levem sine rugis ... scias eum esse litterarum amantem. - Pseudopol. arab. p. 149,15 cuius frons multas rugas habet, eius possessor superbus est. Rasis p. 177, 12 totius corporis macies, rugosa facies = signa hominis malorum morum.

Zu III vgl. Tertullian, de anima c. 5 (= t. I proleg. p. LXX, 1) pavorem ... pallore (sc. anima testatur). - Nemes. de nat. hom. c. 2 (ebenda) $\varphi \circ \beta \circ \cup \mu \varepsilon \vee \eta \varsigma$ (sc. $\tau \eta \varsigma s ~ \psi \cup \chi \eta \xi)$

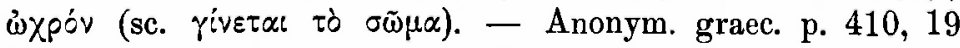

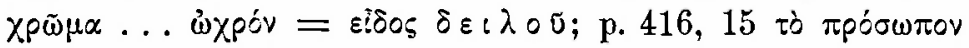

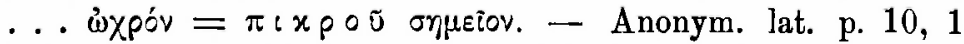
color . . . cum pallore etc. $=$ w e ib i s c h; p. 48,7 circa oculos pallor zeigt einen impudentem, malignum, potentibus inimicum, concinnatorem negotiorum atque causarum, nunquam quietum, nunquam non mali aliquid cogitantem an; p. 107, 1 color . . . pallore deformatus ... imbellem, timidum eundemque tergiversatorem significat, si non aegritudo sit causa palloris. -

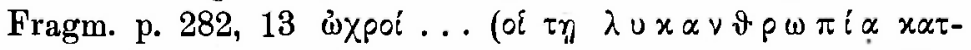

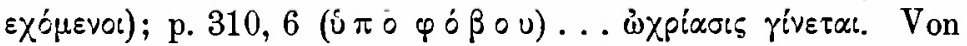
diesen Stellen ruft Anonym. lat. p. 48, 7 die Erinnerung an das Cassiusporträt bei Plutarch, Caesar $62 \dot{\varepsilon} \mu o^{\prime}$. . . oủ $\lambda i \alpha \nu$

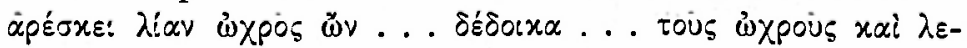
$\pi$ toùs Ėxeívous (= Antonius 11 ; vgl. Brutus $8 \varepsilon \varphi \eta \ldots$.

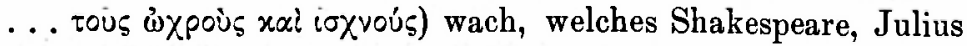
Caesar I 3 mit den Worten: "Yond Cassius has a lean and hungry look. He thinks too much: such men are dangerous ... Would he were fatter . . . Such men as he be never at heart's ease, Whiles they behold a greater than themselves' wiedergibt. Tatsächlich bietet auch der Fanatiker Cassius eine 
nicht ganz unpassende Parallele zu dem acutus sophista religionis agrestis.

\section{Die Völkertypen.}

In das Gebiet der 'Physiognomonia chorographica vel gentilis' gehört die in letzter Linie astrologisch-ethnographische Theorie von den verschiedenen Volksgöttern, welche Julian in seiner Galiläerschrift p. 179, 7 sq. ed. Neumann zur Erklärung

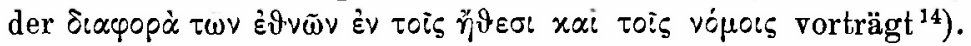
Diese stützt sich nämlich p. 180, 17 auf den Grundsatz tous

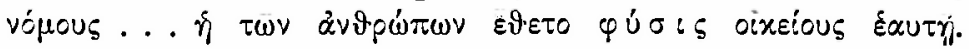

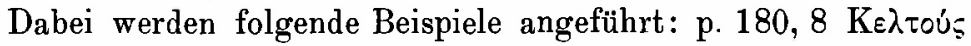

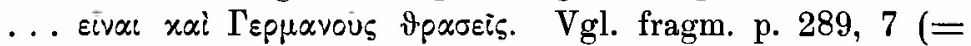

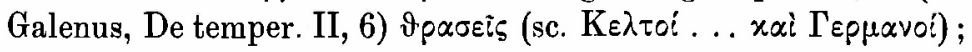
vgl. p. 302,9 .

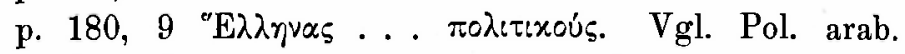
c. XXXV (De Graecis et eorum genere puro) p. 242,9 pulcros ... mores et instituta. Fragm. p. 270, 2 ( $\tau \dot{\delta} \tau \omega \nu{ }^{~} \mathrm{E} \lambda \lambda \gamma_{i} v \omega \nu$

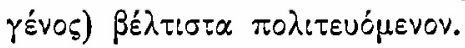

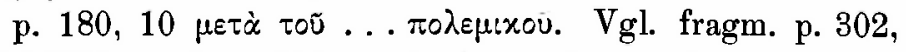

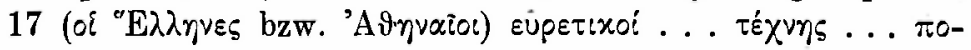
$\lambda \varepsilon \mu\llcorner\times \eta / s$.

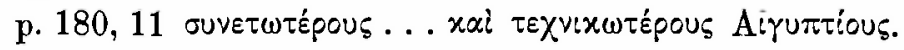
Vgl. Anonym. lat. p. 14, 4 Aegyptii ... callidi, dociles. -

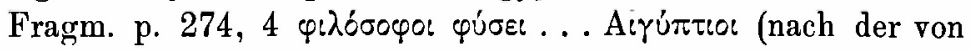
Strabo II, 3, 7 p. 103 bekämpften Lehre des Posidonius) ${ }^{15}$ ).

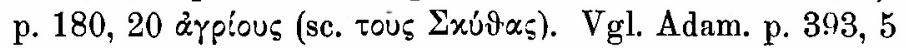

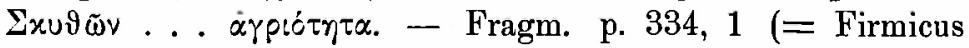
Maternus, Math. I, 1) Scythae ... immani feritatis crudelitate grassantur etc.

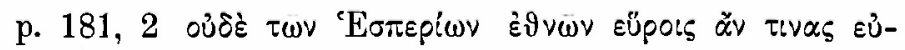

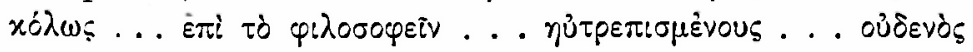

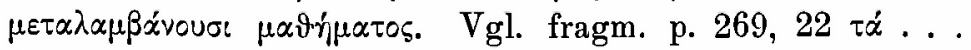

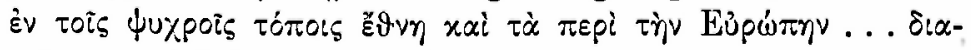

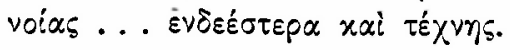

14) S. hierüber unser Programm „Julians Galiäerschrift im Zusammenhang mit seinen übrigen Werken". Freiburg i. B. 1904 S. 13 ff. 15.

15) S. a. a, O. S. 190 . 


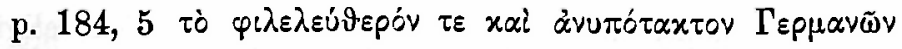

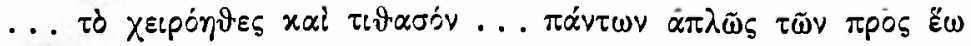

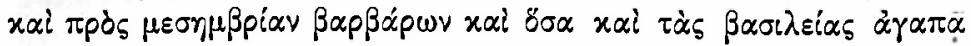

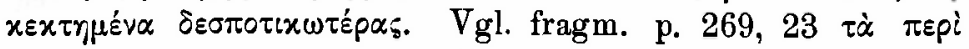

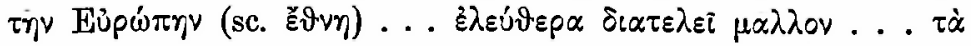

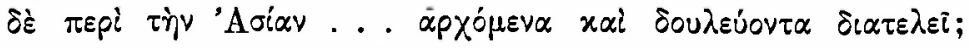

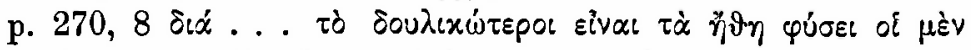

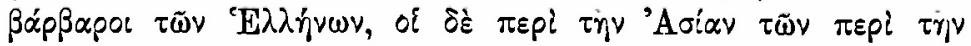

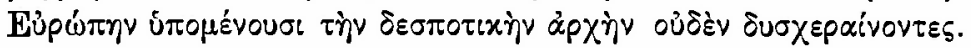

E. D i e Ju d en.

Physiognomonisch sind auch die beiden Attribute, welche Julian, Galiläerschr. p. 201, 12 den Juden beilegt, um ihren störrischen Charakter zu kennzeichnen: Er nennt sie hier $\delta$

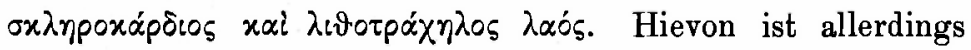
das erstere bereits in der Septuaginta Ez. 3, 7 vertreten; das letztere ist aber ein $\ddot{\alpha} \pi \alpha \xi \lambda \varepsilon \gamma \delta$ $\mu \varepsilon v o v$ und vielleicht von dem Kaiser selbst anstelle des Ex. 33, 3. 5; 34, 9; Deut. 9, 6. 13 und Baruc. 2, 30 gebotenen $\sigma x \lambda \eta p o \tau \rho \alpha ́ x \eta \lambda \circ$ gewählt, das auch im (Cyrill-)Codex Venetus Marcianus 123 (mit übergeschrie-

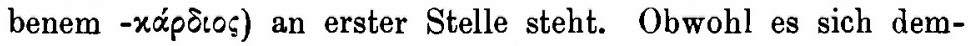
nach hier lediglich um eine Reminiszenz aus dem Septuagintatext handelt, ist es doch nicht uninteressant, daß auch bei den Physiognomonikern derartige Termini vorkommen und von ihnen ganz ubereinstimmend gedeutet werden. $\mathrm{Zu} \sigma x \lambda$ ทpo-

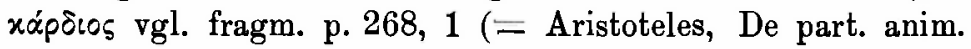

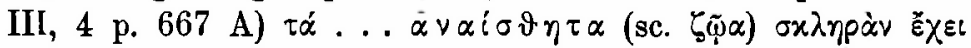

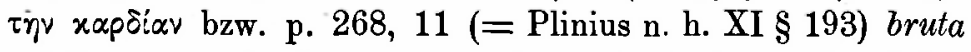
existimantur animalium, quibus durum (cor) riget und $\mathrm{zu} \lambda เ \vartheta^{\circ} \circ-$

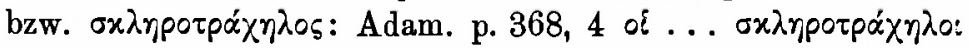
$\bar{\alpha} \mu \alpha \vartheta \varepsilon i \varsigma$ bzw. Anonym. lat. p. 74, 6 cervix dura indocilem hominem ostendit.

Freiburg i. B.

R. Asmus. 